\title{
Health Related Quality of Life and Biochemical Markers for Cachexia Evaluation in Head and Neck Cancer Patients
}

\author{
Joaquim Castro Silva ${ }^{1 *}$ (), Patricia Tavares ${ }^{2}$, Rita Ferreira ${ }^{2}$, Lucio Lara Santos ${ }^{1}$, Eurico Monteiro $^{1}$ \\ ${ }^{1}$ Instituto Português de Oncologia do Porto Francisco Gentil, Porto, Portugal \\ ${ }^{2}$ LAQV, Department of Chemistry, University of Aveiro, Aveiro, Portugal \\ Email: ${ }^{\star}$.castrosilva@hotmail.com
}

How to cite this paper: Castro Silva, J., Tavares, P., Ferreira, R., Santos, L.L. and Monteiro, E. (2021) Health Related Quality of Life and Biochemical Markers for Cachexia Evaluation in Head and Neck Cancer Patients. International Journal of Otolaryngology and Head \& Neck Surgery, 10, 320-335.

https://doi.org/10.4236/ijohns.2021.104029

Received: June 30, 2021

Accepted: July 18, 2021

Published: July 21, 2021

Copyright $\odot 2021$ by author(s) and Scientific Research Publishing Inc. This work is licensed under the Creative Commons Attribution International License (CC BY 4.0).

http://creativecommons.org/licenses/by/4.0/

\begin{abstract}
Cachexia is a multifactorial syndrome related to unintentional weight loss and to loss of muscle and fat mass. In head and neck cancer (HNC) its incidence is important and not only related to a deficient intake of food due to the impact of the disease in the vital functions. A complex disturbance in the normal metabolism of the patient promotes a persistent inflammatory state and a shifting in the metabolism balance toward a catabolic predominance affecting primarily the skeletal muscle. This leads to severe impairment of the functional, emotional and social status and quality of life of the patients that will compromise response to treatment and the disease prognosis. Understanding this deleterious syndrome and mainly identifying it in early stages of the disease is of a major importance in achieving better outcomes to head and neck cancer patients. This study pretends to identify clinical aspects related to cachexia in HNC in a clinical perspective for application on the routine clinical practice. In our study, $30 \mathrm{HNC}$ patients were enrolled and evaluated in terms of nutritional values (actual and loss of weight in the past 6 months, body mass index (BMI), nutritional risk index (NRI), malnutrition universal screening tool), serum biochemical markers (albumin, total proteins, cholesterol, triglycerides, urea, C-reactive protein (CRP), interleukin-6 (IL-6), tumour necrosis factor- $\alpha$ (TNF- $\alpha$ ) and myostatin) and health related quality of life (HRQoL) evaluation (using European Organisation for the Research and Treatment of Cancer (EORTC) quality of life questionnaires (QLQ): EORTC QLQ-C30 and EORTC QLQ-HN43). A minimum follow-up of 48 months was considered for all patients. Our results showed that NRI is a good and sensitive index to identify cachexia. This index uses two parameters, one constitutional (loss of weight) and one biochemical (level of serum albumin). According to this criterion, 16 patients were assigned to the No-cachexia
\end{abstract}


group and 14 patients to the Cachexia group. Significant differences in the constitutional and nutritional values between the two groups were found: the median weight loss was $4.44 \mathrm{~kg}$ in the No-cachexia group and $11.29 \mathrm{~kg}$ in the Cachexia group, while the BMI was 21.88 and 18.33, respectively. In terms of biochemical markers, significant low values of albumin and cholesterol in the Cachexia group were encountered when compared to the No-cachexia group. Regarding the inflammatory and cachexia biomarkers studied, the results show that patients in the Cachexia group had significantly higher levels of CRP and of the proinflammatory cytokines IL- 6 and TNF- $\alpha$ and presented significantly raised levels of the myostatin. In terms of HRQoL evaluation, the scores of the EORTC QLQ-C30 revealed that all the scales and the Summary Score showed lower scores in the Cachexia group, indicating worst quality of life evaluation. The items scores were globally higher in the Cachexia group indicating more important problems related to those items in the Cachexia group. The difference encountered between the groups was significant $(\mathrm{p}<$ 0.001) in all considered scales but two: Dyspnoea and Constipation. Considering the EORTC QLQ-HN43 all the scales and in all single items but one (Wound Healing) the scores were higher in the Cachexia group, indicating a worst degree of problems affecting these group of patients. The difference found between the groups was significant $(\mathrm{p}<0.001)$ in all scales and items but six: Dry Mouth and Sticky Saliva, Skin problems, Problems with Teeth, Trismus, Social Contact and Wound Healing. There were no significant differences in the clinical presentation of the disease between the two groups. The median survival was of 13.5 months in the Cachexia group, significantly lower when compared to the No-cachexia group ( $\mathrm{p}<0.0001$ ), confirming the major impact of cachexia in survival and clinical outcomes in HNC patients. These results of our study show that HRQoL evaluation and serum biochemical markers are sensitive and important tools in identifying and screening cachexia in HNC patients. The methodology followed in this study correlating HRQoL with biochemical markers supports the development of clinical protocols in HNC that include cachexia evaluation. Hopefully this new approach can help to improve prognosis of the disease.

\section{Keywords}

Cachexia, Head Neck Cancer, Quality of Life, Biochemical Markers, Malnutrition

\section{Introduction}

Head and neck cancer (HNC) treatment has evolved through the times, particularly in the last decades with the introduction of the so-called organ preservation treatments either with the use of new drugs or the development of more conservative surgeries using laser or robotics. Nevertheless, besides all this progress, overall survival didn't change much, particularly for larynx cancer and recurrent or metastatic disease [1] [2] [3].

On the other hand, the impact of HNC on the global status of the patients is 
important, contributing to a progressive worsening of the physical, emotional and social conditions with a major impact on their quality of life. Understanding these factors can help us to improve global status of these patients and eventually get a better prognosis for their disease. One of these conditions that impacts the prognosis and quality of life of HNC patients is cachexia [4] [5].

Cachexia is a multifactorial syndrome related with loss of skeletal muscle and fat mass. It's not only, but mostly related with cancer. In HNC its incidence is important and more than $50 \%$ of patients with advanced HNC have important weight loss and cachexia [6].

HNC patients with cachexia present with important unintentional weight loss, asthenia, anorexia, anaemia, fatigue, sarcopenia which led to significant decrease in global functional status.

The establishment of this syndrome considerably affects prognosis of the disease and has a negative impact on the response to treatment, increasing toxicities and complications. This implies a poor overall survival in these patients: cachexia is the cause of death in $20 \%$ of all-cancer patients [6].

The metabolic changes associated with HNC patients are due not only to the limitations imposed by the disease or treatments that limit the adequate intake of nutrients, but the disease itself induces changes at the cellular and molecular level that originate these very metabolic changes leading to a state of cachexia. This multi-factorial paraneoplastic syndrome leads to progressive weight loss and to a permanent inflammatory response.

Weight loss is often observed when the first signs of disease appear and when it reaches $30 \%$ it is generally fatal [6].

The inflammatory response is related to an accentuated catabolism that is mediated by factors released by the tumor and the host. These mediators seem to induce an imbalance between protein synthesis and degradation, leading to protein depletion and, consequently, loss of muscle mass, the degree of which is inversely correlated with the patients' lifespan. In the inflammatory response several biomarkers are implicated: C-reactive protein (CRP), albumin, proteins, interleukin-6 (IL-6), tumor necrosis factor- $\alpha$ (TNF- $\alpha$ ), myostatin and others [7] [8] [9].

Thus, cachexia seems to result from a natural evolution of the oncological disease, regardless of the quantity or quality of food intake.

The cachexia syndrome related to HNC patients is an important factor in these patients and conditioning the prognosis of the disease. HNC patients with cachexia will have worst functional status with impact on their quality of life [5] [6] [7]. Also, these patients will have worst responses to treatment, with lower rates of adhesion to treatment and higher rates of toxicities and complications.

Early identification of cachexia in HNC patients will promote an early intervention in these patients to start a clinical strategy to improve patient status and prevent refractory cachexia that can lead to a better response and adhesion to treatment and reduce rates of toxicities and complications, improving outcomes 
and quality of life.

Quality of life evaluation, body weight and cachexia biomarkers screening can be important tools to the early identifications of patients in risk of cachexia regarding to HNC.

In the literature all these parameters are already studied and their importance in the clinical setting is well proved. Nevertheless, we didn't find any study that has evaluated all these parameters and its inter-relations in the clinical practice in $\mathrm{HNC}$ and we found in recent reviews references to promote these studies in cachexia research [10] [11] [12] [13]. To understand the relations between all these factors and understand their close relation to cachexia in a clinical application perspective was the main purpose of this prospective study.

\section{Material and Methods}

Patients with head and neck squamous cell carcinoma between 2016 and 2017 were evaluated in its first presentation at Department of Otorhinolaryngology of Instituto Português de Oncologia do Porto Francisco Gentil (IPOPFG).

The eligibility criteria included patients: 1 ) no obese (body mass index-BMI $<30$ ) and no diabetic; 2) no prior history of cancer nor submitted to prior cancer treatment.

Body weight, lost of weight in the last 6 months, BMI and Malnutrition Universal Screening Tool (MUST) score were recorded. Serum levels of biochemical markers were determined after blood sample: albumin, total proteins, cholesterol, triglycerides, urea, C-reactive protein, interleukin-6, tumour necrosis factor- $\alpha$ and myostatin.

The blood samples were collected and treated in the same manner of other routine blood tests done in our Institution.

Health Related Quality of life (HRQoL) evaluation was done using European Organisation for the Research and Treatment of Cancer (EORTC) Quality of Life Questionnaires (QLQ): EORTC QLQ-C30 and EORTC QLQ-HN43. These questionnaires are translated and validated for Portuguese and were answered by the patients with the assistance of clinical staff when needed. The Department of Otorhinolaryngology of IPOPFG has implemented since more than a decade routine evaluation of HRQoL for all patients treated.

This methodology was chosen to ensure that all the evaluation and collecting data was taken as it would be on the usual daily clinical routine of the Department and could be reproducible in different clinical Institutions.

This study was approved by the Ethical Committee and all patients signed a consent form.

Thirty males were enrolled with a minimum follow-up of 48 months, and they were assigned into two groups-Cachexia and No-cachexia-according to the Nutritional Index Score.

Values are presented as mean \pm standard deviation for each group. The statistical significance of the differences between groups was determined using the 
unpaired student t-test.

The level of significance was set at $5 \%$ (p-value $<0.05)$. These statistical analyses were performed with the GraphPad Prism ${ }^{\circledR}$ software.

\section{Results}

Thirty patients with HNC were included in this study. All patients were male, with an average age of 55 years (range 43 to 77 years).

After a preliminary analysis of the experimental data from biochemical markers and body weight loss and BMI, patients were redistributed based on the nutrition risk index (NRI) [14] [15].

Nutritional risk was assessed through the application of the NRI tool developed by the Veteran Affair Total Parenteral Cooperative Study Group [16]. This is a tool with high sensitivity and specificity [17] [18]. The NRI is determined using the values of serum albumin, and of the actual and usual percentage weight, based on the following equation:

$\mathrm{NRI}=1.519 \times \mathrm{Alb}$ serum $(\mathrm{g} / \mathrm{dl})+0.417 \times(($ actual weight $/$ usual weight $) \times 100))$

The following criteria were used for the analysis of results: above 100 indicate satisfactory nutritional status; between 97.5 and 100, mild risk of malnutrition; from 83.5 to 97.5 , moderate risk; and below 83.5 , severe risk.

Patients with NRI equivalent to a satisfactory nutritional status or mild risk were considered without cachexia and thus included in the No-cachexia group. Patients with moderate or severe risk were considered with cachexia and included in the Cachexia group.

According to this criteria, 16 patients were assigned to the No-cachexia group and 14 patients to the Cachexia group.

Albumin serum levels were significantly lower (Table 3 ) as were significantly higher the percentage of body weight loss (Table 1) in the Cachexia group. This difference was already expected since these values were used for the determination of NRI (criteria to define the two groups). Regarding BMI, this was lower in patients in the Cachexia group $(\mathrm{p}<0.01)$. Patients from No-cachexia group presented an average BMI of 22, which is an indicator of healthy weight [19].

Patients were screened also using the MUST score and we observe that in the No-cachexia group most of the subjects presented with a MUST score of 0 . On the other hand, in the Cachexia group 25\% of patients were identified at high risk of malnutrition, presented a MUST score of 4 (Table 1).

No significant age differences were noticed between groups $(54.9 \pm 7.3$ on Cachexia group and $58.3 \pm 9.3$ on No-cachexia group). There were also no significant differences between the clinical presentation of the tumours between the two groups. In Cachexia group all patients presented with TNM stage IV tumours and most of them in the pharynx: 5 patients in the oropharynx, 1 in the nasopharynx, 7 in the hypopharynx and 1 in the oral cavity; in No-cachexia group most of the patients presented locoregionally advanced tumours (12 patients with TNM stage IV and 3 patients with TNM stage III) and only 1 pre- 
sented with a TNM stage I tumour and also most of these tumours were located in pharynx: 3 in the oropharynx, 1 in the nasopharynx, 4 in the hypopharynx, 5 in the larynx and 3 in the oral cavity (Table 2).

A minimum follow-up of 48 months was considered to all patients and overall survival was recorded. A median survival of 13.5 months in the Cachexia group was significantly lower when compared to the No-cachexia group $(\mathrm{p}<0.0001)$ and survival comparison between the groups is shown by the Kaplan-Meir

Table 1. Characterization of patients relative to NRI, age, body weight loss, BMI and MUST score.

\begin{tabular}{ccc}
\hline & \multicolumn{2}{c}{ Groups } \\
\cline { 2 - 3 } Cachexia & No-cachexia \\
\hline NRI & $86.90 \pm 7.31^{* * * *}$ & $104.60 \pm 3.12$ \\
Age (years) & $54.9 \pm 7.3$ & $58.3 \pm 9.3$ \\
Body weight loss (Kg) & $11.29 \pm 6.08^{* *}$ & $4.44 \pm 3.87$ \\
BMI (Kg/m ( $^{2}$ & $18.33 \pm 2.96^{* *}$ & $21.88 \pm 3.53$ \\
MUST (n) & 1 & 9 \\
0 & 3 & 4 \\
1 & 5 & 2 \\
2 & 1 & 0 \\
4 & 4 & 16 \\
\hline TOTAL & 14 & \\
\hline
\end{tabular}

Values are expressed as mean \pm standard deviation. ${ }^{* *} \mathrm{p}<0.01{ }^{* * * *} \mathrm{p}<0.0001$.

Table 2. Characterization of patients relative to location and TNM stage of the disease.

\begin{tabular}{ccc}
\hline & \multicolumn{2}{c}{ Groups } \\
Location $(\mathrm{n})$ & Cachexia & No-cachexia \\
\cline { 2 - 3 } Oral cavity & 1 & 3 \\
Nasopharynx & 1 & 1 \\
Oropharynx & 5 & 3 \\
Hypopharynx & 7 & 4 \\
Larynx & 0 & 5 \\
TOTAL & 14 & 16 \\
\hline TNM Stage (n) & & 1 \\
I & 0 & 0 \\
II & 0 & 3 \\
III & 0 & 12 \\
IV & 14 & 16 \\
\hline TOTAL & 14 & \\
\hline
\end{tabular}


graphic (Figure 1).

Concerning biochemical parameters assessed in serum samples, albumin levels were significantly lower in the Cachexia group $(\mathrm{p}<0.0001)$, already expected since these values were used in NRI calculation and, consequently, in patients' assessment to each group. A significant decrease in cholesterol levels $(\mathrm{p}<0.001)$ was observed in the Cachexia group (Table 3).

For the remaining biochemical parameters assessed (glucose, urea, total proteins and triglycerides), no differences were observed between groups (Table 3 ).

Regarding to the inflammatory and cachexia biomarkers studied, the results show that patients in the Cachexia group had significantly higher levels of CRP and of the proinflammatory cytokines IL- 6 and TNF- $\alpha$ and presented significantly elevated levels of the myostatin, which may be suggestive of muscle mass loss in this group of patients (Table 3 ).

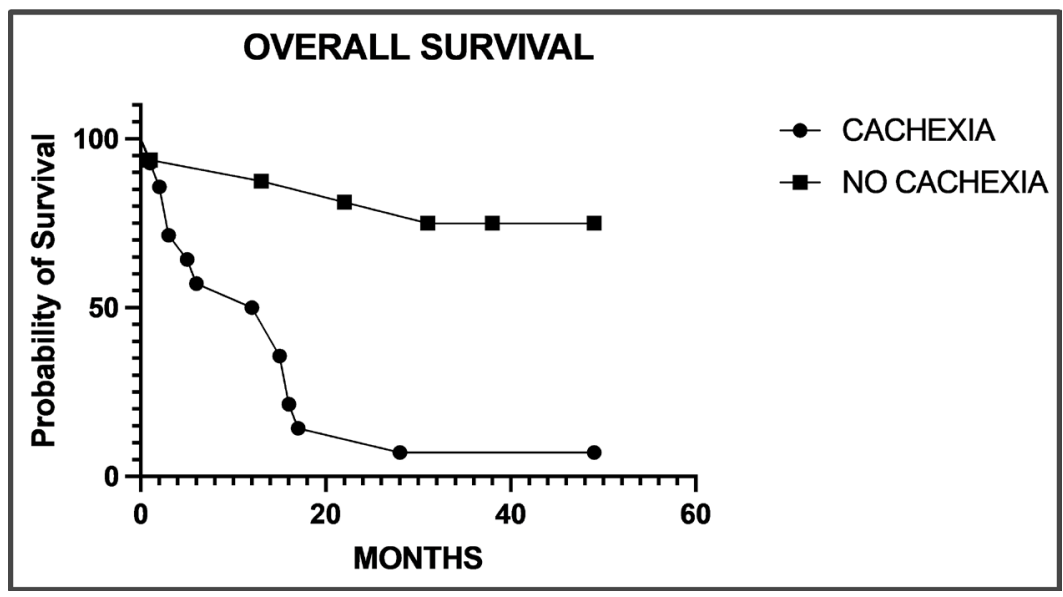

Figure 1. Overall survival (Kaplan-Meir).

Table 3. Characterization of patients relative to serum biochemical parameters.

\begin{tabular}{ccc}
\hline & \multicolumn{2}{c}{ Groups } \\
\cline { 2 - 3 } & Cachexia & No-cachexia \\
\hline Albumin $(\mathrm{g} / \mathrm{L})$ & $34.21 \pm 1.49^{* * * *}$ & $43.25 \pm 0.66$ \\
Glucose $(\mathrm{mmol} / \mathrm{L})$ & $5.521 \pm 0.217$ & $5.531 \pm 0.203$ \\
Total Proteins (g/L) & $72.14 \pm 2.163$ & $72.81 \pm 1.108$ \\
Urea (mmol/L) & $3.336 \pm 0.291$ & $4.939 \pm 0.248$ \\
Cholesterol (mmol/L) & $3.653 \pm 0.205^{* * *}$ & $4.931 \pm 0.248$ \\
Triglycerides (mmol/L) & $1.114 \pm 0.092$ & $1.334 \pm 0.178$ \\
\hline CRP (mg/L) & $49.79 \pm 10.73^{* *}$ & $691.5 \pm 60.94$ \\
IL-6 (OD) & $856.5 \pm 65.41^{*}$ & $482.7 \pm 80.66$ \\
TNF- $\alpha(\mathrm{OD})$ & $681.9 \pm 72.43^{* *}$ & $504.7 \pm 34.11$ \\
\hline Myostatin (OD) & $700.7 \pm 57.02^{* *}$ & \multicolumn{2}{c}{139} \\
\hline
\end{tabular}

Values are expressed as mean \pm standard deviation. ${ }^{*} \mathrm{p}<0.05 ;{ }^{* *} \mathrm{p}<0.01 ;{ }^{* * *} \mathrm{p}<0.001 ; * * * \mathrm{p}<0.0001$. OD arbitrary units of optical density. (from immunoblot analysis) 
All patients underwent quality of life evaluation answering at first time observation to the EORTC Quality of life Questionnaire C-30 (EORTC QLQ-C30) [20] and the updated EORTC Head and Neck Quality of life Questionnaire module HN43 (EORTC QLQ-HN43) [21] [22].

EORTC QLQ-C30 has 30 questions that are combined in 13 scales: Global Quality of Life, Physical Functioning, Role Functioning, Social Functioning, Emotional Functioning, Cognitive Functioning, Fatigue, Pain, Nausea and Vomiting, Dyspnoea, Sleeping Disturbances, Appetite loss, Constipation, Diarrhoea and Financial Impact. Also, there is a global score, the EORTC-QLQ-C30 Summary Score [23], that was calculated. All the scales and the Summary Score showed lower scores in the Cachexia group, indicating worst quality of life evaluation. The items scores were globally higher in the Cachexia group indicating more important problems related to those items in the Cachexia group. The difference encountered between the groups was significant $(p<0.001)$ in all considered scales but two: Dyspnoea and Constipation (Table 4).

EORTC QLQ-HN43 module has 43 questions and is combined into 12 scales (Fear of Progression, Body Image, Dry Mouth and Sticky Saliva, Pain in the Mouth, Sexuality, Problems with Senses, Problems with Shoulder, Skin Problems, Social Eating, Speech, Swallowing and Problems with Teeth) and 7 single items (Coughing, Swelling in the Neck, Neurological Problems, Trismus, Social Contact, Weight Loss and Wound Healing). In all the scales and in all single

Table 4. EORTC QLQ C-30 scores.

\begin{tabular}{|c|c|c|c|}
\hline Scales and items & Cachexia & No-cachexia & $\mathrm{p}$ \\
\hline Global health status/QoL & $42.27 \pm 35.12$ & $64.07 \pm 23.51$ & $<0.001$ \\
\hline Physical functioning & $70.41 \pm 20.10$ & $87.09 \pm 13.21$ & $<0.001$ \\
\hline Role functioning & $75.00 \pm 25.11$ & $87.50 \pm 18.76$ & $<0.001$ \\
\hline Emotional functioning & $72.03 \pm 19.51$ & $84.38 \pm 15.77$ & $<0.001$ \\
\hline Cognitive functioning & $82.15 \pm 25.71$ & $89.59 \pm 15.96$ & 0.010 \\
\hline Social functioning & $73.81 \pm 31.16$ & $93.75 \pm 14.75$ & $<0.001$ \\
\hline Fatigue & $40.48 \pm 30.07$ & $17.37 \pm 16.22$ & $<0.001$ \\
\hline Nausea and vomiting & $2.33 \pm 18.20$ & $3.13 \pm 12.50$ & 0.010 \\
\hline Pain & $36.91 \pm 20.86$ & $23.96 \pm 24.32$ & $<0.001$ \\
\hline Dyspnoea & $11.91 \pm 28.06$ & $12.50 \pm 16.67$ & 0.797 \\
\hline Sleeping Disturbances & $47.62 \pm 38.60$ & $31.25 \pm 30.96$ & $<0.001$ \\
\hline Appetite loss & $35.72 \pm 42.29$ & $18.75 \pm 29.74$ & $<0.001$ \\
\hline Constipation & $9.52 \pm 24.21$ & $6.25 \pm 13.44$ & 0.199 \\
\hline Diarrhoea & $7.14 \pm 26.73$ & $0.00 \pm 0.00$ & 0.010 \\
\hline Financial difficulties & $45.24 \pm 46.42$ & $20.84 \pm 26.88$ & $<0.001$ \\
\hline Summary Score & $74.45 \pm 13.60$ & $85.38 \pm 11.09$ & $<0.001$ \\
\hline
\end{tabular}

Values are expressed as mean \pm standard deviation. 
items but one (Wound Healing) the scores were higher in the Cachexia group, indicating a worst degree of problems affecting these group of patients. The difference found between the groups was significant $(p<0.001)$ in all scales and items but six: Dry Mouth and Sticky Saliva, Skin problems, Problems with Teeth, Trismus, Social Contact and Wound Healing (Table 5).

\section{Discussion}

Patients with HNC have high rates of morbidity and mortality that are closely associated with critical weight loss, a common feature in these patients [24].

When compared to other cancers, HNC patients have greater weight loss incidence, in part due to the difficulty in swallowing presented by these patients [25].

Also, in these patients, smoking and drinking habits, the most common etiological factors associated to the development of HNC, are important and have been associated to malnutrition [25]. Some studies indeed showed that the risk of malnutrition increases with the amount of alcohol consumed [26].

On the other hand, several studies associate HNC patients with cachexia and showed that this syndrome was not reverted by parental nutrition [27].

Table 5. EORTC QLQ HN-43 scores.

\begin{tabular}{|c|c|c|c|}
\hline Scales and items & Cachexia & No-cachexia & $\mathrm{p}$ \\
\hline Fear of progression & $44.05 \pm 21.29$ & $34.38 \pm 23.15$ & $<0.001$ \\
\hline Body image & $42.86 \pm 32.57$ & $21.53 \pm 18.80$ & $<0.001$ \\
\hline Dry mouth \& sticky saliva & $25.00 \pm 20.41$ & $22.92 \pm 20.97$ & 0.298 \\
\hline Pain in the mouth & $42.86 \pm 27.90$ & $20.31 \pm 19.71$ & $<0.001$ \\
\hline Sexuality & $36.91 \pm 31.37$ & $19.79 \pm 25.98$ & $<0.001$ \\
\hline Problems with senses & $17.86 \pm 27.32$ & $7.2975 \pm 10.49$ & $<0.001$ \\
\hline Problems with shoulder & $19.05 \pm 31.25$ & $8.33 \pm 10.54$ & $<0.001$ \\
\hline Skin problems & $4.76 \pm 10.42$ & $4.16 \pm 7.99$ & 0.513 \\
\hline Social eating & $24.41 \pm 24.56$ & $11.98 \pm 22.56$ & $<0.001$ \\
\hline Speech & $30.95 \pm 28.24$ & $19.17 \pm 14.17$ & $<0.001$ \\
\hline Swallowing & $33.33 \pm 26.75$ & $21.88 \pm 24.13$ & $<0.001$ \\
\hline Problems with teeth & $22.22 \pm 28.24$ & $18.75 \pm 15.03$ & 0.136 \\
\hline Coughing & $35.71 \pm 35.72$ & $25.00 \pm 22.77$ & $<0.001$ \\
\hline Swelling in the neck & $42.86 \pm 40.15$ & $22.92 \pm 26.44$ & 0.362 \\
\hline Neurological problems & $19.05 \pm 25.20$ & $6.25 \pm 13.44$ & 0.551 \\
\hline Trismus & $21.43 \pm 30.96$ & $18.75 \pm 29.74$ & $<0.001$ \\
\hline Social contact & $9.52 \pm 24.21$ & $8.33 \pm 14.91$ & $<0.001$ \\
\hline Weight loss & $50.00 \pm 31.35$ & $18.75 \pm 24.25$ & 0.001 \\
\hline Wound healing & $2.38 \pm 8.91$ & $6.25 \pm 13.44$ & $<0.001$ \\
\hline
\end{tabular}

Values are expressed as mean \pm standard deviation. 
The evidence shows that clinical management of cachexia is difficult and complex and poorly recognized or assessed [14] [28]. The cause of this can be in part justified by a misdiagnosis of cachexia in head and neck cancer patients. So, in our study a special focus was put in the definition of the criteria to differentiate patients with cachexia and without cachexia.

Although loss of weight or percentage of lost weight in the past 6 months, and even MUST scores, showed differences between patients it was the Nutritional Risk Index that showed high sensitivity to compare the patients in terms of with or without a wasting phenotype when analysed with the biochemical markers. The NRI was the index chosen because it is highly sensitive when compared to other nutritional assessments. Studies showed that when investigating the ability to detect conditions such as sarcopenia, pre-cachexia, and cancer cachexia, NRI presented the better accuracy (about 74.7\%) in identifying patients with at least one of these conditions, when compared to other screening tools (e.g. MST, Malnutrition Screening Tool; MUST, Malnutrition Universal Screening Tool) [14].

In this way, our study confirms NRI as a good indicator and screening tool to evaluate cachexia in the context of HNC patients. This tool uses values related to the nutritional and constitutional aspects of the patients: the percentage of weight lost, and a biochemical marker of depletion, serum albumin. We can say that NRI addresses a good compromise between these two factors so important in the definition of cachexia.

Once established the experimental groups by the nutritional criteria above defined, we've enrolled two groups of patients that are homogeneous in terms of demographic and clinical patterns. We have only enrolled males to avoid differences due to a different hormonal environment in women. There were no differences between ages. In terms of clinical presentation and staging of the disease there were no asymmetries between the two groups. As expected, all of the patients in the Cachexia group presented with a locoregional advanced disease-TNM stage IV, but we manage to have a similar group of patients in the No-cachexia group, with only one patient with early stage disease-TNM stage I. In terms of location of the tumour there were also few asymmetries between the groups: eventually a higher proportion of pharynx cancers in the Cachexia group and a higher proportion of larynx cancers in the No-cachexia group. This can be explained in terms of a bigger impact of the pharynx tumours in the intake and swallow of food when compared to larynx tumours, even when considered locally advanced tumours.

With the two groups well defined, we've done a minimum follow-up of 48 months and encountered a significant difference in overall survival between the groups. In the Cachexia group only one patient in the fourteen considered is still alive and conclude the treatment proposed, all the others died from the disease with a median survival of 13.5 months and most of them didn't complete the treatment proposed or didn't have the conditions for elective treatment. In the 
No-cachexia group most of the patients completed the proposed treatment and overall survival was similar to the overall survival for HNC patients. Our study confirms that cachexia and nutritional impairment has a negative impact in prognosis and survival for HNC patients.

Cachexia is a multifactorial syndrome that is responsible for the presence of an inflammatory state and a catabolic environment in the patients. These features are expressed in the high levels of some biomarkers in serum samples from these patients. The presence of this Inflammation state is a major characteristic of this syndrome and distinguishes it from other syndromes like sarcopenia [29] [30]. One the other hand inflammation is always present in head and neck cancer patients, even if not at very high levels [31]. So, another point in our study was to demonstrate the relation between inflammation and body weight loss and cachexia in head and neck cancer patients.

In our study we found a significant difference in the serum levels of CRP, IL-6 and TNF-a between our two groups of patients. We can assume that HNC patients with cachexia have a significant level of inflammation when compared to patients without cachexia.

CRP, although not specific, is a sensitive marker of inflammation and it is synthesized in the liver induced by proinflammatory cytokines, mainly IL-6. The persistence of this inflammatory state leads to a continuous production of this acute phase protein, promoting the reprioritization of amino acid metabolism in peripheral tissues, especially muscles, towards the liver. Therefore, muscle catabolism occurs. Our study showed an increase of circulating CRP levels in the group of Cachexia patients and was paralleled by the increase of IL-6, which is known to promote the liver synthesis of CRP [32] [33].

Besides that, TNF- $\alpha$ high serum levels were previously associated with HNC [34]. We also found in our study a significantly high level of TNF- $\alpha$ in the Cachexia group confirming once more the relationship of inflammation with the wasting phenotype.

On the other hand, studies demonstrated IL- 6 and TNF- $\alpha$ are related to cellular pathways that mediate muscle mass loss and protein synthesis inhibition [35] [36]. Myostatin is secreted and expressed mainly by skeletal muscle cells and has a main role in the regulation of muscle mass, blocking myoblast proliferation and suppression of satellite cell activation [37]. So, there is a negative correlation between elevated myostatin serum levels and skeletal muscle mass and studies have demonstrated that myostatin is involved in various diseases involving muscle mass loss [38] [39] [40]. Our data shows that patients in Cachexia group have significant higher levels of myostatin when compared to patients in the No-cachexia group. So myostatin is an important marker related to skeletal muscle mass loss and can signalling cachexia.

Health related quality of life evaluation has an important role in the management of HNC patients. This evaluation addresses issues that consider the patient as an individuum and its relations in terms of himself, his family and society 
where he lives. This evaluation is well established and accurate and changes verified, in the settle of HNC, among others, are related to changes in terms of the disease [20] [41] [42] [43]. EORTC has developed validated questionnaires and recently have made an actualization of the specific questionnaire module for head and neck cancer, the EORTC QLQ-HN43 [21] [22]. In our study we have applied to all patients at presentation both questionnaires proposed by EORTC to evaluate head and neck cancer patients: the EORTC QLQ-C30 with the newest EORTC QLQ-HN43. Our data showed that most of the scales and items were related to significantly worst quality of life parameters in the patients of the Cachexia group. The Global QoL and the Summary Score for EORTC QLQ-C30 can be global measures of HRQL [23] [44] [45] for these patients and show significant differences when considering cachexia patients. Scores for Dyspnoea and constipation scales didn't show significant differences and we can say that these items aren't important in the settle of cachexia when considering HNC patients.

The newly specific head and neck cancer module, the EORTC QLQ-HN43, also gave significant differences in the scores of most of the scales and items, demonstrating worst scores in the Cachexia patients group. The presence of a non-significant difference in the scores between the two groups in the scales and items Dry Mouth and Sticky Saliva, Problems with Teeth, Trismus, can be considered that these problems affect all HNC patients and eventually can show that cachexia in HNC patients is not, at least in part, related exclusively to the limitation of eating and swallowing.

These results from our study support the usefulness of HRQoL in the evaluation and follow-up of HNC patients and can be important in the identification of cachexia in these patients.

In resume our study developed a methodology with significatively results in terms of nutritional parameters, biochemical markers and HRQoL scores in the evaluation of cachexia in the clinical context of HNC that can be used in on a routine clinical practice, contributing to fill a gap seen in the research in this field that can help improving prognosis and HRQoL in HNC patients.

\section{Conclusions}

Cachexia is a deleterious condition and has a negative impact on prognosis of HNC patients. Overall survival of patients with cachexia is very low with a median overall survival of 13.5 months in our study.

Cachexia patients present with significant weight loss and significantly high levels of serum biomarkers of inflammation and muscle wasting like CRP, IL-6, TNF-a or myostatin, and significantly lower levels of nutritional serum markers like albumin or cholesterol.

NRI is an important screening tool to identify cachexia in head and neck cancer patients.

HRQoL evaluation, using validated questionnaires like the EORTC QLQ-C30 and EORTC QLQ-HN43, is an important tool in the management of HNC can- 
cer patients and can identify early deterioration of the global or specific conditions of HNC patients.

The implementation of clinical protocols using these tools, NRI, serum biomarkers (albumin, CRP, IL-6, TNF-a, myostatin) and HRQoL evaluation, can promote an early identification of cachexia in HNC patients leading to a strategical clinical approach to control this negative condition and improve patients' prognosis.

\section{Conflicts of Interest}

The authors declare no conflicts of interest regarding the publication of this paper.

\section{References}

[1] Johnson, D.E., Burtness, B., Leemans, C.R., et al. (2020) Head and Neck Squamous Cell Carcinoma. Nature Reviews Disease Primers, 6, 92.

https://doi.org/10.1038/s41572-020-00224-3

[2] Du, E., et al. (2019) Long-Term Survival in Head and Neck Cancer: Impact of Site, Stage, Smoking, and Human Papillomavirus Status. Laryngoscope, 129, 2506-2513. https://doi.org/10.1002/lary.27807

[3] Pulte, D., et al. (2010) Changes in Survival in Head and Neck Cancers in the Late 20th and Early 21st Century: A Period Analysis. Oncologist, 15, 994-1001. https://doi.org/10.1634/theoncologist.2009-0289

[4] Orell-Kotikangas, H., et al. (2017) Cachexia at Diagnosis Is Associated with Poor Survival in Head and Neck Cancer Patients. Acta Oto-Laryngologica, 137, 778-785. https://doi.org/10.1080/00016489.2016.1277263

[5] Lis, C.G., et al. (2012) Role of Nutritional Status in Predicting Quality of Life Outcomes in Cancer-A Systematic Review of the Epidemiological Literature. Nutrition Journal, 11, 27. https://doi.org/10.1186/1475-2891-11-27

[6] Couch, M., et al. (2007) Cancer Cachexia Syndrome in Head and Neck Cancer Patients: Part I. Diagnosis, Impact on Quality of Life and Survival, and Treatment. Head Neck, 29, 401-411. https://doi.org/10.1002/hed.20447

[7] Couch, M.E., et al. (2015) Cancer Cachexia Update in Head and Neck Cancer: Definitions and Diagnostic Features. Head and Neck, 37, 594-604. https://doi.org/10.1002/hed.23599

[8] Fearon, K.C.H., et al. (2012) Cancer Cachexia: Mediators, Signaling, and Metabolic Pathways. Cell Metabolism, 16, 153-166. https://doi.org/10.1016/j.cmet.2012.06.011

[9] Loumaye, A. and Thissen, J.-P. (2017) Biomarkers of Cancer Cachexia. Clinical Biochemistry, 50, 1281-1288. https://doi.org/10.1016/j.clinbiochem.2017.07.011

[10] Berardi, E., Madaro, L., Lozanoska-Ochser, B., et al. (2021) A Pound of Flesh: What Cachexia Is and What It Is Not. Diagnostics (Basel), 11, 116.

https://doi.org/10.3390/diagnostics11010116

[11] Dev, R. (2019) Measuring Cachexia-Diagnostic Criteria. Annals of Palliative Medicine, 8, 24-32. https://doi.org/10.21037/apm.2018.08.07

[12] McDonald, J.J., Fallon, M.T. and Laird, B.J.A. (2019) Meaningful Measures in Cancer Cachexia: Implications for Practice and Research. Current Opinion in Supportive and Palliative Care, 13, 323-327. 
https://doi.org/10.1097/SPC.0000000000000472

[13] Castillo-Martínez, L., Castro-Eguiluz, D., et al. (2018) Nutritional Assessment Tools for the Identification of Malnutrition and Nutritional Risk Associated with Cancer Treatment. Revista de investigación clínica, 70, 121-125. https://doi.org/10.24875/RIC.18002524

[14] Bhuachalla, É.B.N., et al. (2018) Computed Tomography Diagnosed Cachexia and Sarcopenia in 725 Oncology Patients: Is Nutritional Screening Capturing Hidden Malnutrition? Journal of Cachexia, Sarcopenia and Muscle, 9, 295-306. https://doi.org/10.1002/jcsm.12258

[15] Aziz, E.F., et al. (2011) Malnutrition as Assessed by Nutritional Risk Index Is Associated with Worse Outcome in Patients Admitted with Acute Decompensated Heart Failure: An ACAP-HF Data Analysis. Heart International, 6, e2.

https://doi.org/10.4081/hi.2011.e2

[16] Veterans Affairs Total Parenteral Nutrition Cooperative Study Group (1991) Perioperative Total Parenteral Nutrition in Surgical Patients. The New England Journal of Medicine, 325, 525-532. https://doi.org/10.1056/NEJM199108223250801

[17] Buzby, G.P., et al. (1988) A Randomized Clinical Trial of Total Parenteral Nutrition in Malnourished Surgical Patients: The Rationale and Impact of Previous Clinical Trials and Pilot Study on Protocol Design. The American Journal of Clinical Nutrition, 47, 357-365. https://doi.org/10.1093/ajcn/47.2.357

[18] Thieme, R.D., et al. (2013) Nutritional Risk Index Is Predictor of Postoperative Complications in Operations of Digestive System or Abdominal Wall? Arquivos Brasileiros de Cirurgia Digestiva, 26, 286-292. https://doi.org/10.1590/S0102-67202013000400007

[19] World Health Organization (2021) Body Mass Index BMI. http://www.euro.who.int/en/health-topics/disease-prevention/nutrition/a-healthy-li festyle/body-mass-index-bmi

[20] Aaronson, N., et al. (1993) The European Organization for Research and Treatment of Cancer QLQ-C30: A Quality-of-Life Instrument for Use in International Clinical Trials in Oncology. Journal of the National Cancer Institute, 85, 365-376. https://doi.org/10.1093/jnci/85.5.365

[21] Singer, S., et al. (2015) Measuring Quality of Life in Patients with Head and Neck Cancer: Update of the EORTC QLQ-H\&N Module, Phase III. Head Neck, 37, 1358-1367. https://doi.org/10.1002/hed.23762

[22] Singer, S., et al. (2019) International Validation of the Revised European Organisation for Research and Treatment of Cancer Head and Neck Cancer Module, the EORTC QLQ-HN43: Phase IV. Head Neck, 41, 1725-1737. https://doi.org/10.1002/hed.25609

[23] Giesinger, J., et al. (2016) Replication and Validation of Higher Order Models Demonstrated That a Summary Score for the EORTC QLQ-C30 Is Robust. Journal of Clinical Epidemiology, 69, 79-88. https://doi.org/10.1016/j.jclinepi.2015.08.007

[24] Jager-Wittenaar, H., et al. (2007) Critical Weight Loss in Head and Neck Cancer-Prevalence and Risk Factors at Diagnosis: An Explorative Study. Support Care Cancer, 15, 1045-1050. https://doi.org/10.1007/s00520-006-0212-9

[25] Righini, C.-A., et al. (2013) Assessment of Nutritional Status at the Time of Diagnosis in Patients Treated for Head and Neck Cancer. European Annals of Otorhinolaryngology, Head and Neck Diseases, 130, 8-14. https://doi.org/10.1016/j.anorl.2012.10.001

[26] Martín Villares, C., et al. (2004) Heavy Alcohol Intake, Malnutrition and Head and 
Neck Cancer Patients. Nutricion Hospitalaria, 19, 348-352.

[27] Jager-Wittenaar, H., et al. (2017) High Prevalence of Cachexia in Newly Diagnosed Head and Neck Cancer Patients: An Exploratory Study. Nutrition, 35, 114-118. https://doi.org/10.1016/j.nut.2016.11.008

[28] Nishikawa, D., et al. (2018) The Impact of Skeletal Muscle Depletion on Head and Neck Squamous Cell Carcinoma. ORL; Journal for Oto-Rhino-Laryngology and Its Related Specialties, 80, 1-9. https://doi.org/10.1159/000485515

[29] Ali, S. and Garcia, J.M. (2014) Sarcopenia, Cachexia and Aging: Diagnosis, Mechanisms and Therapeutic Options. Gerontology, 60, 294-305.

https://doi.org/10.1159/000356760

[30] Liguori, I., et al. (2018) Sarcopenia: Assessment of Disease Burden and Strategies to Improve Outcomes. Clinical Interventions in Aging, 13, 913-927. https://doi.org/10.2147/CIA.S149232

[31] Charles, K.A., et al. (2016) Systemic Inflammation Is an Independent Predictive Marker of Clinical Outcomes in Mucosal Squamous Cell Carcinoma of the Head and Neck in Oropharyngeal and Non-Oropharyngeal Patients. BMC Cancer, 16, 124. https://doi.org/10.1186/s12885-016-2089-4

[32] Mahmoud, F.A. and Rivera, N.I. (2002) The Role of C-Reactive Protein as a Prognostic Indicator in Advanced Cancer. Current Oncology Reports, 4, 250-255. https://doi.org/10.1007/s11912-002-0023-1

[33] Duffy, S.A., et al. (2008) Interleukin-6 Predicts Recurrence and Survival among Head and Neck Cancer Patients. Cancer, 113, 750-757. https://doi.org/10.1002/cncr.23615

[34] Andersson, B.-Å., et al. (2014) Plasma Tumor Necrosis Factor- $\alpha$ and C-Reactive Protein as Biomarker for Survival in Head and Neck Squamous Cell Carcinoma. Journal of Cancer Research and Clinical Oncology, 140, 515-519. https://doi.org/10.1007/s00432-014-1592-8

[35] Yoshida, T., et al. (2013) Molecular Mechanisms and Signaling Pathways of Angiotensin II-Induced Muscle Wasting: Potential Therapeutic Targets for Cardiac Cachexia. The International Journal of Biochemistry \& Cell Biology, 45, 2322-2332. https://doi.org/10.1016/j.biocel.2013.05.035

[36] Visser, M., et al. (2002) Relationship of Interleukin-6 and Tumor Necrosis Factor-Alpha with Muscle Mass and Muscle Strength in Elderly Men and Women: The Health ABC Study. The Journals of Gerontology. Series A, Biological Sciences and Medical Sciences, 57, M326-M332. https://doi.org/10.1093/gerona/57.5.M326

[37] Manole, E., et al. (2018) Myokines as Possible Therapeutic Targets in Cancer Cachexia. Journal of Immunology Research, 2018, Article ID: 8260742. https://doi.org/10.1155/2018/8260742

[38] Magee, T.R., et al. (2006) Myostatin Short Interfering Hairpin RNA Gene Transfer Increases Skeletal Muscle Mass. The Journal of Gene Medicine, 8, 1171-1181. https://doi.org/10.1002/jgm.946

[39] García, P.S., et al. (2010) Brief-Reports: Elevated Myostatin Levels in Patients with Liver Disease: A Potential Contributor to Skeletal Muscle Wasting. Anesthesia \& Analgesia, 111, 707-709. https://doi.org/10.1213/ANE.0b013e3181eac1c9

[40] White, T.A. and LeBrasseur, N.K. (2014) Myostatin and Sarcopenia: Opportunities and Challenges-A Mini-Review. Gerontology, 60, 289-293.

https://doi.org/10.1159/000356740

[41] Velikova, G., et al. (2012) Health-Related Quality of Life in EORTC Clinical Tri- 
als-30 Years of Progress from Methodological Developments to Making a Real Impact on Oncology Practice. European Journal of Cancer-Supplement, 10, 141-149. https://doi.org/10.1016/S1359-6349(12)70023-X

[42] Bjordal, K., et al. (1999) Quality of Life in Head and Neck Cancer Patients: Validation of the European Organization for Research and Treatment of Cancer Quality of Life Questionnaire-H\&N35. Journal of Clinical Oncology, 17, 1008-1019.

https://doi.org/10.1200//CO.1999.17.3.1008

[43] Bjordal, K. and Kaasa, S. (1992) Psychometric Validation of the EORTC Core Quality of Life Questionnaire, 30-Item Version and a Diagnosis-Specific Module for Head and Neck Cancer Patients. Acta Oncologica, 31, 311-321.

https://doi.org/10.3109/02841869209108178

[44] Husson, O., et al. (2020) The EORTC QLQ-C30 Summary Score as Prognostic Factor for Survival of Patients with Cancer in the "Real-World": Results from the Population-Based PROFILES Registry. Oncologist, 25, e722-e732. https://doi.org/10.1634/theoncologist.2019-0348

[45] Kasper, B. (2020) The EORTC QLQ-C30 Summary Score as a Prognostic Factors for Survival of Patients with Cancer: A Commentary. Oncologist, 25, e610-e611. https://doi.org/10.1634/theoncologist.2019-0749 